\title{
Optimization of Liquid Culture Condition of a Novel Fungus Hygrophoropsis sp. and Antioxidant Activity of Extracts
}

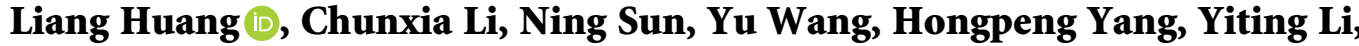 \\ and Litong Ban (10) \\ College of Agronomy and Resources Environment, Tianjin Agricultural University, Tianjin 300384, China \\ Correspondence should be addressed to Litong Ban; banlitong@126.com
}

Received 2 August 2019; Revised 21 June 2020; Accepted 30 June 2020; Published 1 August 2020

Academic Editor: Saad Tayyab

Copyright $\odot 2020$ Liang Huang et al. This is an open access article distributed under the Creative Commons Attribution License, which permits unrestricted use, distribution, and reproduction in any medium, provided the original work is properly cited.

\begin{abstract}
To evaluate pharmacological activities of a novel fungus Hygrophoropsis sp., the influence of aeration rate on the production of mycelial biomass, exopolysaccharides (EPS), and intrapolysaccharides (IPS) in the fungus Hygrophoropsis sp. was investigated. And the water extracts of cultured Hygrophoropsis sp. mycelia and the fermentation broth were analyzed for their antioxidation activity by using four different assay methods such as hydroxyl radical scavenging, superoxide radical scavenging, hydrogen peroxide scavenging, and reducing power. The Hygrophoropsis sp. was cultivated under various aeration rates in a 71 bioreactor. The highest mycelial biomass $(3.98 \mathrm{mg} / \mathrm{mL})$ and IPS production $(19.63 \mathrm{mg} / \mathrm{g})$ were obtained at aeration rate $4.5 \mathrm{v} . \mathrm{v} . \mathrm{m}$. The results showed that Hygrophoropsis sp., in general, possesses a strong antioxidation activity in all assays tested. The deproteinized extracts had stronger antioxidation activity as compared to the un-deproteinized extracts by using superoxide radical scavenging, hydrogen peroxide scavenging, and reducing power. Besides, the un-deproteinized extracts had stronger antioxidation activity as compared to the deproteinized extracts by using hydroxyl radical scavenging. Thus, the polysaccharide extractions from the Hygrophoropsis sp. studied have antioxidant activities in vitro, which may be a good source of natural antioxidants or further investigation as potential natural antioxidants.
\end{abstract}

\section{Introduction}

Some synthetic antioxidants, such as butylated hydroxyanisole and butylated hydroxytoluene, are used for food additives but not in medical health because of their toxicity [1]. Mushrooms are increasingly attractive as natural, nontoxic antioxidants and as potential sources of physiologically important components [2-4]. Polysaccharides are an important component of various types of fungi, a major factor in their bioactive properties, such as the suppression of damage caused by cytostatics and radiation to the immune system cells [5-8].

A novel fungus called "Weimo" in Ninghe District Tianjin Province was found among reeds. The fruiting body is yellow, like butter (Figure 1). This wild mushroom is rare but tasty, which was sold for 4000 RMB per kilogram. Mycelia isolated from fruiting body tissues were cultured and preserved on slant medium in our laboratory. It was identified as Hygrophoropsis sp. belonging to Hygrophoropsidaceae family by ITS sequence.

This edible fungus is hard to cultivate for fruiting bodies but only the right environment and special substance are available. However, fungal mycelia can easily be acquired in sufficient quantities by fermentation in order to produce biologically active supplements, and the submerged cultivation method is an acceptable method [4].

In comparison with the commercial mushroom products, mycelia or fermentation broth has many advantages such as shorter culture period, consistent product quality, and independent of seasonality [9-11]. Hygrophoropsis sp. mycelia were obtained by submerged cultivation in a batch system from shaking flask to fermenter. The in vitro antioxidative activities of the extracts were tested by using different assay methods. The biological activities of the crude polysaccharides suggested it is applicable for resources development. 


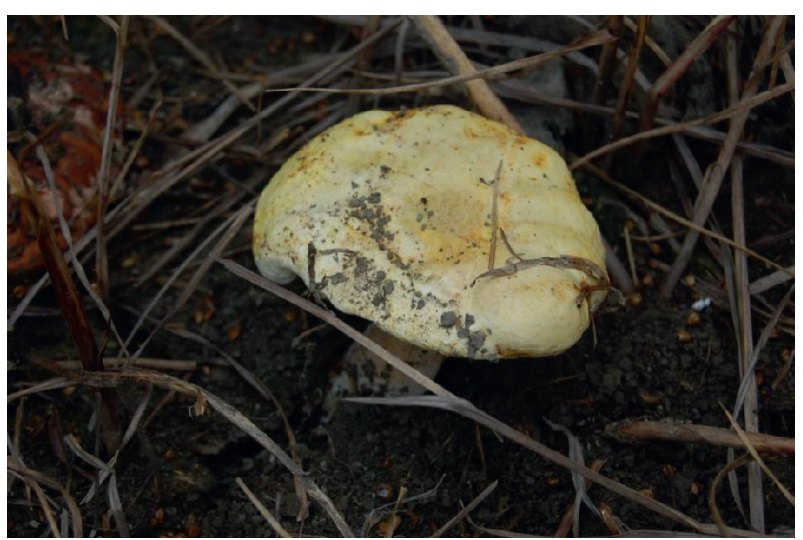

FIgURE 1: Wild fungus "Weimo" among reeds.

\section{Materials and Methods}

The fruiting body of "Weimo" was picked in Ninghe District, Tianjin province. Cultured mycelia were obtained from tissues and preserved at Technology Research and Development Center for Edible Fungus, Tianjin Agricultural University, Tianjin, China.

2.1. Preparation of Liquid Medium. The mycelia was cultured in the liquid medium with $95 \mathrm{~mL}$ working volume in $250 \mathrm{~mL}$ triangular flask at $28^{\circ} \mathrm{C}$, which consisted of $15 \mathrm{mg} / \mathrm{mL}$ glucose, $10 \mathrm{mg} / \mathrm{mL}$ sucrose, and $4 \mathrm{mg} / \mathrm{mL}$ yeast powder.

2.2. Liquid Fermentation. The $4 \mathrm{~L}$ liquid fermentation medium was added to a $7 \mathrm{~L}$ fermenter which has been already sterilized. Then, the medium was sterilized at $121^{\circ} \mathrm{C}$ for $20 \mathrm{~min}$ again. After the strain seed culture was added, the aeration rate was set at $3,4.5$, and $6 \mathrm{~L} / \mathrm{min}$, respectively. The mycelia were collected at different intervals. Samples were centrifuged at $5000 \mathrm{rpm}$ for 10 minutes; the supernatant and precipitate were preserved, respectively, for polysaccharides determination.

\subsection{Isolation of IPS and EPS}

2.3.1. Extraction of IPS. The extraction method of polysaccharides was performed according to the procedure described by Zhang et al. [12], with some modifications. The IPS solution was extracted from dried mycelia filtered by hot water with 1:10 ratio, which was precipitated by adding 4 times of ethanol (90\%) subsequently. The precipitate was suspended in water and centrifuged to remove insoluble material.

2.3.2. Extraction of EPS. The supernatant was concentrated to $1 / 2$ of the original volume by rotary evaporation under reduced pressure at $55^{\circ} \mathrm{C}$. The concentrated EPS solution was treated with 4 times of ethanol and then centrifuged at $5000 \mathrm{rpm}$ for $10 \mathrm{~min}$ to get the precipitate. The precipitate was suspended in water and centrifuged to remove insoluble material [13].
2.4. Purification of Polysaccharides. Deproteinization procedure was carried out according to reference [14] with some modifications as follows: The crude polysaccharides solution was added 4 times the volume of savage reagent (chloroform: normal butanol =5:1), shaken violently for $30 \mathrm{~min}$, and then rested for $10 \mathrm{~min}$. The intermediate protein layer was removed from the mixture which was centrifuged for $10 \mathrm{~min}$. Moreover, the supernatant solution was preserved. The procedure was repeated 3-5 times to ensure that all proteins are removed.

Un-deproteinized polysaccharides were purified without deproteinization procedure but further procedure [15], while deproteinized polysaccharides were subsequently purified as follows: the supernatant solution was added 4 times the volume of ethanol and kept for $12 \mathrm{~h}$ at $4^{\circ} \mathrm{C}$; the precipitation was the purified polysaccharides after centrifuging at $5000 \mathrm{rpm}$ for $10 \mathrm{~min}$. Furthermore, polysaccharides were washed with ethanol for 2-3 times, and then lyophilized; the polysaccharides sample was obtained finally.

2.5. Determination of the Glucose Standard Curve. The yield of polysaccharides was determined by phenol-sulfuric acid method as in reference [16] with some modifications. The standard glucose solution was prepared (the glucose was dried to the constant weight) with a concentration of $0.04 \mathrm{mg} \mathrm{mL}^{-1}$. The glucose solution was taken as 0 (reference), $0.2,0.3,0.4,0.5,0.6,0.7$, and $0.8 \mathrm{~mL}$, respectively, which were complemented to $1.0 \mathrm{~mL}$ with distilled water. Then, they were shaken after adding phenol (5\%) $0.5 \mathrm{~mL}$ and sulfuric acid $2.5 \mathrm{~mL}$. The absorbance was measured at $490 \mathrm{~nm}$ after 30 minutes at room temperature. The standard curve was drawn with the polysaccharides concentration as the horizontal coordinate and the absorbance value as the ordinate.

The polysaccharides sample $(1 \mathrm{~mL})$ substituted for the glucose solution was determined according to the above method.

2.6. Extraction and Optimization of IPS. The IPS was extracted with water extracting-alcohol precipitating. The IPS ( $1 \mathrm{~g}$ ) was extracted by rotating evaporator, and the supernatant was used for the determination. The orthogonal experiment of $\mathrm{L}_{9}\left(3^{3}\right)$ was designed with the solid-liquid ratio, extraction time, and extraction temperature as the factors. The levels of the factors are shown in Table 1.

2.6.1. Calculation of Extraction Rate of Polysaccharides. The extraction rate of polysaccharides was calculated as follows. The extraction rate of polysaccharides $(\%)=X \times V /$ $M \times 100 \%$, where $X$ is sample polysaccharides concentration $(\mathrm{mg} / \mathrm{mL}), V$ is sample volume $(\mathrm{mL})$, and $M$ is mass of mycelia $(g)$.

2.6.2. Alcohol Precipitation of Polysaccharides. Aqueous polysaccharides of $5 \mathrm{~mL}$ were added with ethanol to the final concentration of ethanol solution $60 \%, 65 \%, 70 \%, 75 \%, 80 \%$, $85 \%$, and $90 \%(\mathrm{v} / \mathrm{v})$ and sat quietly for $24 \mathrm{~h}$. The optimum 
TABLE 1: Orthogonal factors and levels.

\begin{tabular}{lcccc}
\hline Independent variables & Coded & \multicolumn{3}{c}{ Levels } \\
& symbols & 1 & 2 & 3 \\
\hline Solid-liquid ratio & $\mathrm{A}$ & $1: 30$ & $1: 40$ & $1: 50$ \\
Extraction time $(\mathrm{h})$ & $\mathrm{B}$ & 1 & 2 & 3 \\
Extraction temperature $\left({ }^{\circ} \mathrm{C}\right)$ & $\mathrm{C}$ & 85 & 90 & 95 \\
\hline
\end{tabular}

ethanol concentration was obtained by the content of polysaccharides.

\subsection{Determination of Antioxidant Capacity}

2.7.1. Ability of Scavenging Hydroxyl Radicals. The ability of scavenging hydroxyl radicals of sample polysaccharides was determined according to the principle of the Fenton reaction system as in reference [17] with some modifications. The reaction system consisted of phosphate buffer solution $(\mathrm{pH}$ 7.4) $0.5 \mathrm{~mL}$, Saffron solution (0.5\%) $0.1 \mathrm{~mL}$, EDTA Na $\mathrm{Fe}^{2+}$ solution $(0.1 \mathrm{~mol} / \mathrm{L}) 0.5 \mathrm{~mL}$, sample polysaccharides $3.5 \mathrm{~mL}$, and $\mathrm{H}_{2} \mathrm{O}_{2}(6 \%) 0.4 \mathrm{~mL}$. The absorbance was measured at $520 \mathrm{~nm}$ after a water bath at $40^{\circ} \mathrm{C}$ for $30 \mathrm{~min}$. The blank was done as follows: sample polysaccharides solution was replaced by distilled water of equal volume, and the control group, polysaccharides solution, and EDTA $\mathrm{Na}_{2}-\mathrm{Fe}^{2+}$ solution were replaced in the same way. The scavenging rate of hydroxyl radicals was calculated as follows: Hydroxyl radicals scavenging rate $(\%)=\left(1-\left(\Delta A_{520}\right.\right.$ of sample $-\Delta A_{520}$ of blank $) /\left(\Delta A_{520}\right.$ of control $-\Delta A_{520}$ of blank $\left.)\right) \times 100 \%$.

2.7.2. Ability of Scavenging Superoxide Anion Radicals. The ability of scavenging superoxide anion free radicals $\left(\mathrm{O}^{2-}\right)$ was determined according to the principle of pyrogallol autooxidation [18]. Each sample of polysaccharide solution with different concentrations $(0.2 \mathrm{~mL})$ was mixed with Tris- $\mathrm{HCl}$ ( $\mathrm{pH} 8.2$ ) buffer solution $(5.6 \mathrm{~mL})$. Then, pyrogallol solution (preheat at $25^{\circ} \mathrm{C}, 30 \mathrm{mmol} / \mathrm{L}, 0.2 \mathrm{~mL}$ ) was added after the water bath at $25^{\circ} \mathrm{C}$ for $10 \mathrm{~min}$. The reaction of the mixture was accurate $30 \mathrm{~min}$ after shaking vigorously. Finally ascorbic acid $\left(V_{C}\right)$ solution $(5 \%, 0.1 \mathrm{~mL})$ was added to terminate the reaction. The absorbance was measured at $420 \mathrm{~nm}$ after resting for $10 \mathrm{~min}$. In control group, $0.2 \mathrm{~mL}$ distilled water was used to replace the sample, and in blank group, $5.6 \mathrm{~mL}$ Tris- $\mathrm{HC} 1$ solution was used to replace the pyrogallol solution. The scavenging rate of superoxide anion radicals was calculated as follows: Superoxide anion radicals scavenging rate $(\%)=\left(\left(\Delta A_{420}\right.\right.$ of control $-\Delta A_{420}$ of sample $) /$ $\Delta A_{420}$ of control) $\times 100 \%$.

2.7.3. Reducing Power. The Oyaizu [19] method was used to determine the reduction force of the sample polysaccharides with minor modifications. The different concentrations of polysaccharides solution $(1 \mathrm{~mL})$ were mixed with phosphate buffer solution ( $\mathrm{pH} 6.6,2.5 \mathrm{~mL}$ ) and $1 \%$ potassium ferricyanide solution $(2.5 \mathrm{~mL})$. The mixture was shaken and centrifuged for $10 \mathrm{~min}$ at $800 \mathrm{rpm}$. The supernatant $(2.5 \mathrm{~mL})$ was mixed with distilled water and $0.1 \%$ ferric chloride solution $(2.5 \mathrm{~mL}$, respectively). The absorbance was measured at $700 \mathrm{~nm}$ against a blank after shaking and resting for $10 \mathrm{~min}$.

2.7.4. Ability of Scavenging 1, 1-Diphenyl-2-picrylhydrazyl (DPPH) Radicals. DPPH free radical scavenging activity was measured according to the method of Kao and Chen [20], with some modifications. The polysaccharides solution with different concentrations $(3 \mathrm{~mL})$ was mixed with $\mathrm{DPPH}$ solution $(0.004 \%$ in ethanol, $1.0 \mathrm{~mL})$. The mixture was shaken vigorously and rested for $30 \mathrm{~min}$ in the dark, and the absorbance was then measured at $517 \mathrm{~nm}$ against the blank. $V_{\mathrm{C}}$ was used for positive control. The scavenging rate of $\mathrm{DPPH}$ radicals was calculated as follows: $\mathrm{DPPH}$ radicals scavenging rate $(\%)=\left(\left(\Delta A_{517}\right.\right.$ of blank $-\Delta A_{517}$ of sample $) /$ $\Delta A_{517}$ of blank $) \times 100 \%$.

2.8. Statistical Analysis. Statistical analysis involved the use of the statistical analysis system SPSS v.17. 0 program. Analysis of variance was performed by ANOVA procedures. Each item of data represents mean values and standard deviation (SD) of three replicates. Significant differences between the two means were determined by Tukey's test. $P$ values $<0.05$ were regarded as significant.

\section{Results}

3.1. Glucose Standard Curve. The glucose standard curve is shown in Figure 2. $R$-square is 0.9989 , which revealed good linear relation between absorbance and glucose solution concentration.

\subsection{Effect of Aeration Rate on the Intracellular Polysaccharide} (IPS) and Exopolysaccharide (EPS) Yield. The effect of aeration rate on IPS and EPS production is shown in Figure 3. The yield of IPS production was higher than that of EPS. The yield of IPS was the highest $(19 \mathrm{mg} / \mathrm{g})$ at $40 \mathrm{~h}$ with the aeration rate $4.5 \mathrm{~L} / \mathrm{min}$, while EPS production reached the highest rate $(0.056 \mathrm{mg} / \mathrm{mL})$ at $40 \mathrm{~h}$ with the aeration rate $6 \mathrm{~L} /$ min.

\subsection{Optimization of Extraction Conditions for IPS Production.} The range $R$ is applied to estimate the magnitude of influence of each parameter [21]. According to the results of orthogonal analysis in Table 2, the magnitude of influence for the extraction of IPS from Weimo mycelia can be sorted by $A$ (Solid-liquid ratio) $>B$ (Extraction time) $>C$ (Extraction temperature). There were significant differences among the three factors on the extraction of IPS from Weimo mycelia (Table 3 ) and in contrast to $R$ values (Table 2), it can be found that the parameter solid-liquid ratio is the most significant parameter for the extraction of IPS from Weimo mycelia, and extraction temperature is the least important parameter.

The optimal value of specific parameter can be determined based on the results of $\mathrm{K}_{i}$ in Table 2. In the present research, the optimum values for each parameter are as follows: $A=1: 30, B=2, C=85$. Under the optimum 


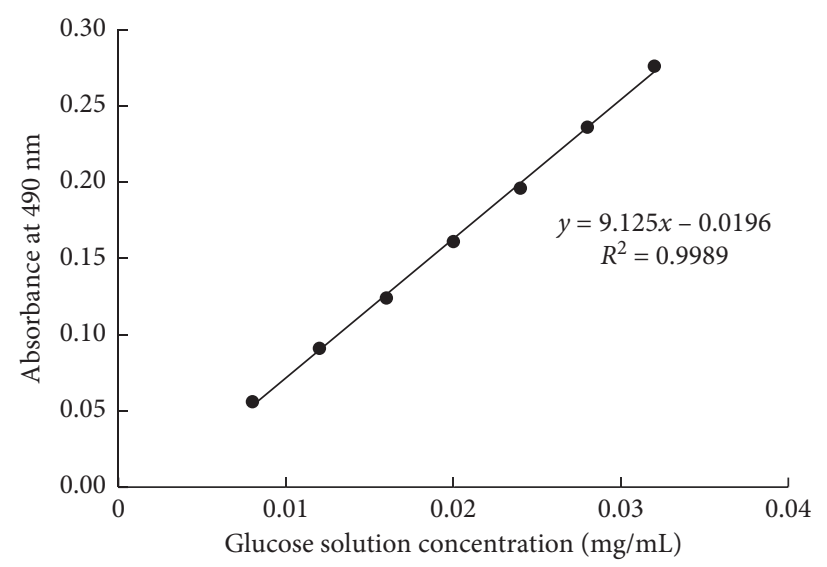

Figure 2: Glucose standard curve.

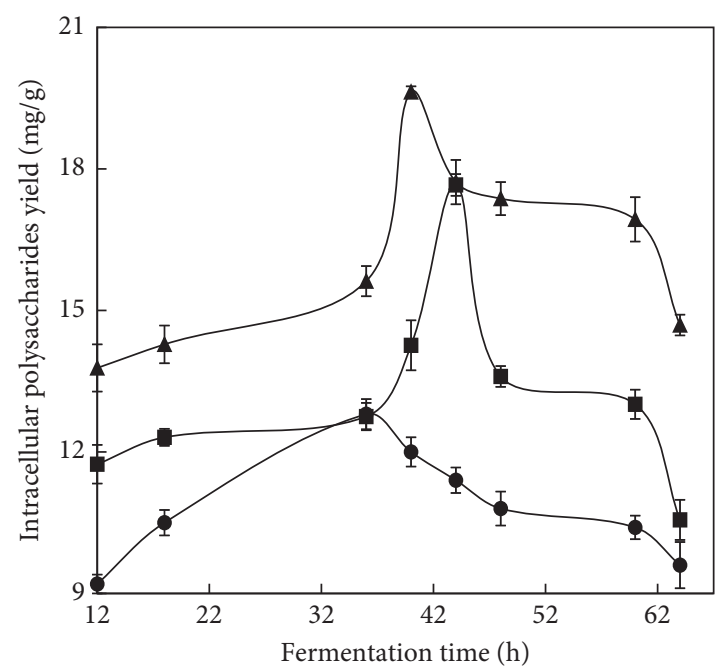

(a)

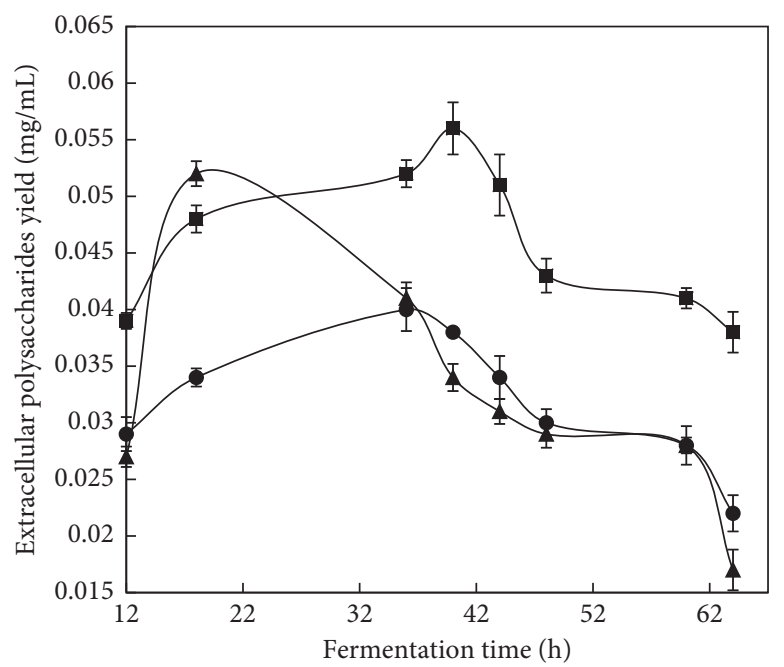

(b)

FIGURE 3: Time profiles of (a) intracellular polysaccharide (IPS) yield and (b) exopolysaccharide (EPS) yield in Hygrophoropsis sp. using a 7-1 stirred tank reactor at different aeration rates; $(\bullet) 3 \mathrm{~L} / \mathrm{min} ;(\boldsymbol{\Delta}) 4.5 \mathrm{~L} / \mathrm{min} ;(\mathbf{\square}) 6 \mathrm{~L} / \mathrm{min}$. Each value is a mean of three biological replicates.

TABLE 2: IPS extraction rates of the orthogonal test with different levels of the parameter.

\begin{tabular}{lcccc}
\hline Test number & $A$ & $B(\mathrm{~h})$ & $C\left({ }^{\circ} \mathrm{C}\right)$ & IPS extraction rate $(\%)$ \\
\hline 1 & $1: 30$ & 1 & 85 & 11.26 \\
2 & $1: 30$ & 2 & 90 & 10.48 \\
3 & $1: 30$ & 3 & 95 & 8.72 \\
4 & $1: 40$ & 1 & 90 & 8.19 \\
5 & $1: 40$ & 2 & 95 & 8.69 \\
6 & $1: 40$ & 3 & 85 & 8.40 \\
7 & $1: 50$ & 1 & 95 & 7.48 \\
8 & $1: 50$ & 2 & 85 & 9.18 \\
9 & $1: 50$ & 3 & 90 & 6.89 \\
$K_{1}$ & 10.15 & 8.98 & 9.61 & \\
$K_{2}$ & 8.43 & 9.45 & 8.52 & \\
$K_{3}$ & 7.85 & 8 & 8.30 & \\
$R$ & 2.3 & 1.45 & 1.31 & \\
\hline
\end{tabular}

$K_{i}$ : the mean values of index for the factors at each level and subscript $i$ denotes the specific level of the parameter, $i=1,2,3 . R$ : the difference between maximum $K_{i}$ and minimum $K_{i}$ for the corresponding parameter, as follows: $R=K_{i} \max -K_{i} \min , i=1,2,3$. extraction method (A1B2C1) of IPS based on the orthogonal optimization method, the extraction rate of IPS was $11.36 \%$. The extraction rate and yield of IPS were higher than those of group $A 1 B 1 C 1$, which validates the reliability of design method and the optimization strategy for IPS extraction that is proposed in the present research.

3.4. Effect of Ethanol Concentration on the Precipitation of Polysaccharides. The yield of polysaccharides in supernatant gradually decreased, and the yield of polysaccharides in precipitate increased gradually with the increase of ethanol concentration (Figure 4 ). When the concentration of ethanol was in the range of $80-85 \%$, the yield of polysaccharides in supernatant decreased, instead, and the yield of polysaccharides in precipitation increased obviously. The yield of polysaccharides in supernatant and precipitate tended to be stable at $85-90 \%$. However, the yield of polysaccharides in precipitation continued to increase, when ethanol concentration reached $95 \%$. On the premise of ensuring maximum 
TABLE 3: Variance analysis for IPS extraction rates.

\begin{tabular}{lccccc}
\hline Source of variation & Quadratic sum & Degree of freedom & Mean square & $F$ value & Significance level \\
\hline Solid-liquid ration & 8.60 & 2 & 4.30 & 87.08 & 0.01 \\
Extraction time & 3.26 & 2 & 1.63 & 33.07 & 30.04 \\
Extraction temperature & 2.97 & 2 & 0.03 & & 0.03 \\
Deviation & 0.10 & 2 & & & \\
Total & 14.92 & & & \\
\hline
\end{tabular}

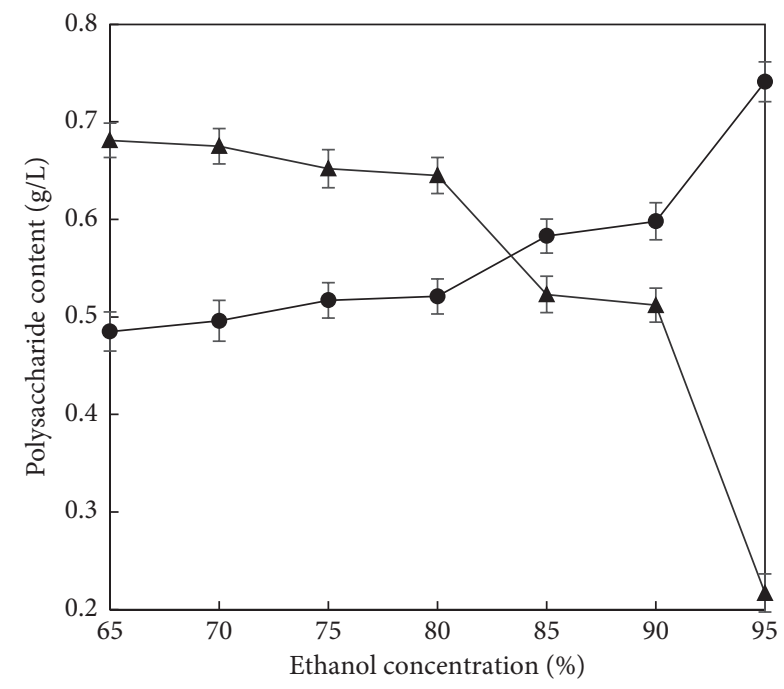

FIGURE 4: Effect of ethanol concentration on polysaccharides precipitation; ( $)$ polysaccharides in precipitation; $(\mathbf{A})$ polysaccharides in supernatant. Each value is a mean of three biological replicates.

precipitation of polysaccharides and saving reagents, $80-85 \%$ ethanol was selected for experiment. Within this range, the yield of polysaccharides in precipitation was $0.52-0.58 \mathrm{mg} / \mathrm{mL}$, as the yield of polysaccharides in supernatant was $0.65-0.52 \mathrm{mg} / \mathrm{mL}$.

\subsection{Determination of Antioxidant Activity}

\subsubsection{Scavenging Ability of Hydroxyl Radicals}

(1) Scavenging Ability of Hydroxyl Radicals on IPS. The scavenging ability of hydroxyl radicals on un-deproteinized IPS was stronger than the deproteinized IPS (Figure 5). When the content of un-deproteinized IPS was at $0.05-0.1 \mathrm{mg} / \mathrm{mL}$, the scavenging rate of hydroxyl radicals increased significantly from $3.21 \%$ to $9.56 \%$. The scavenging rate of hydroxyl radicals was significantly different when the content of un-deproteinized IPS and deproteinized IPS was at $0.1-0.4 \mathrm{mg} / \mathrm{mL}$. With regard to un-deproteinized IPS, the scavenging rate was from $9.56 \%$ to $11.67 \%$ within the above concentration range, while the scavenging rate of deproteinized IPS was from $7.39 \%$ to $10.12 \%$. The scavenging rate of hydroxyl radicals increased little in the range of $0.1-0.4 \mathrm{mg} / \mathrm{mL}$ un-deproteinized IPS, and the maximum scavenging rate of hydroxyl radicals reached $11.67 \%$ at the content of un-deproteinized IPS $0.4 \mathrm{mg} / \mathrm{mL}$. Compared with

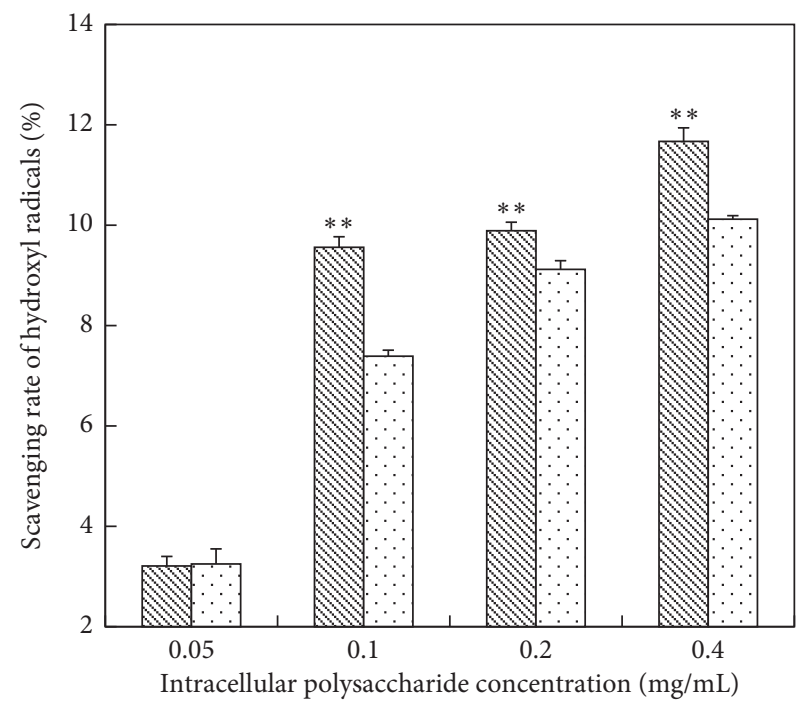

FIGURE 5: Scavenging hydroxyl radical activity of IPS; (圆) undeproteinized IPS; (回) deproteinized IPS. The significance in difference between un-deproteinized IPS and deproteinized IPS was calculated by the paired-samples $T$ test $\left({ }^{* *} P<0.01\right)$.

VC solution, both of the un-deproteinized IPS and deproteinized IPS were lower than VC solution in scavenging hydroxyl radicals. When $\mathrm{VC}$ solution was at $0.4 \mathrm{mg} / \mathrm{mL}$, the scavenging rate of hydroxyl radicals reached $47 \%$, which was far more than the scavenging rate of two kinds of polysaccharides solution at $0.4 \mathrm{mg} / \mathrm{mL}$ (Table 4 ). With regard to the deproteinized IPS, the scavenging rate of hydroxyl radicals increased gradually during the enrichment of crude polysaccharides. The scavenging rate of hydroxyl radicals was $10.12 \%$ when the content of deproteinized IPS was $0.4 \mathrm{mg} / \mathrm{mL}$.

(2) Scavenging Ability of Hydroxyl Radicals on EPS. With regard to EPS, there is no significant difference between the un-deproteinized EPS and deproteinized EPS. However, the scavenging rate of un-deproteinized EPS was slightly higher than deproteinized EPS (Figure 6). The scavenging ability of hydroxyl radicals strengthened with the increasing concentrations of EPS (Figure 6). It is obvious that the scavenging rate tended to be stable when the concentrations of EPS were from 8 to $10 \mathrm{mg} / \mathrm{mL}$. In addition, the maximum scavenging rate (the un-deproteinized EPS) was $47.1 \%$ while that of the deproteinized was $45.9 \%$. Both of un-deproteinized EPS and deproteinized EPS, by contrast, were lower than VC solution in scavenging hydroxyl radicals. When VC 
TABLE 4: Scavenging hydroxyl radical activity of VC.

\begin{tabular}{lccccc}
\hline Scavenging rate of hydroxyl radical scavenging (\%) & \multicolumn{4}{c}{ VC concentration $(\mathrm{mg} / \mathrm{mL})$} \\
& 0.1 & 0.2 & 0.4 & 0.6 & 0.8 \\
\hline VC & $33.3 \pm 0.88$ & $44.7 \pm 0.65$ & $47 \pm 0.78$ & $49 \pm 0.08$ & $50.1 \pm 016$ \\
\hline
\end{tabular}

Each value is a mean of three biological replicates. Data shown are mean \pm standard deviation.

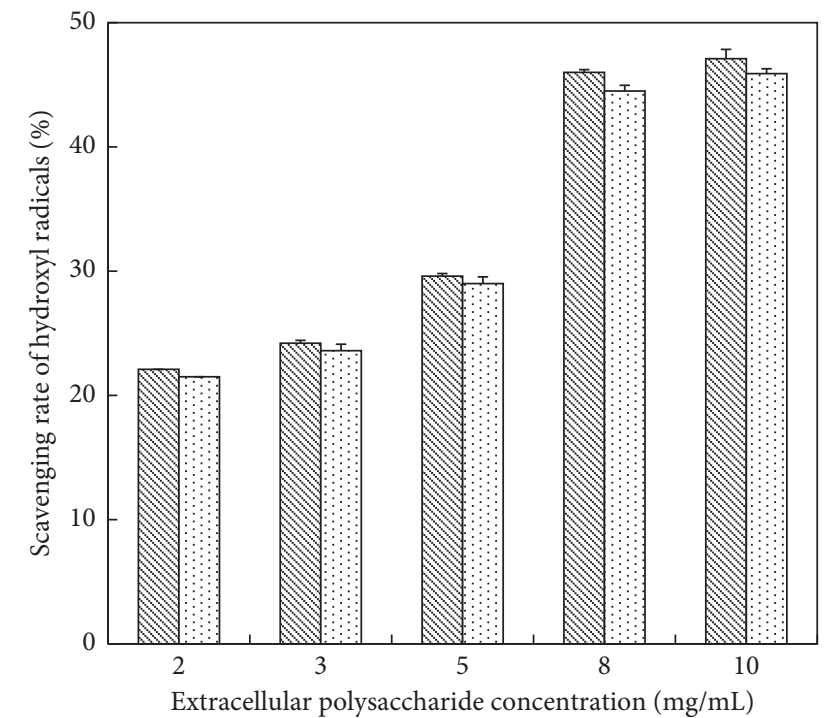

FIGURE 6: Scavenging hydroxyl radical activity of EPS; (专) undeproteinized EPS; ([.) deproteinized EPS. The significance in difference between un-deproteinized EPS and deproteinized EPS was calculated by the paired-samples $T$ test $\left({ }^{* *} P<0.01\right)$.

solution was at $0.8 \mathrm{mg} / \mathrm{mL}$, the scavenging rate had reached $50.1 \%$, while when the un-deproteinized EPS was at $10 \mathrm{mg} /$ $\mathrm{mL}$, the scavenging rate was $47.1 \%$.

\subsubsection{Scavenging Ability of Superoxide Anion Radicals}

(1) Scavenging Ability of Superoxide Anion Radicals on IPS. The scavenging ability of un-deproteinized IPS on superoxide anion free radicals was significantly weaker than that of the deproteinized (Figure 7). When the concentration of the IPS was $0.4 \mathrm{mg} / \mathrm{mL}$, the scavenging rate of superoxide anion radicals (un-deproteinized IPS) was $29.98 \%$, while the scavenging rate of deproteinized IPS was 51 . 34\%. As a whole, the scavenging rate of VC solution was higher than un-deproteinized IPS. The scavenging rate of VC solution was $40.5 \%$ (Table 5) when VC concentration was at $0.4 \mathrm{mg}$ / $\mathrm{mL}$. While the scavenging rate of deproteinized IPS was $29.98 \%$ at the same concentration. With regard to deproteinized IPS, the scavenging rate $(51.34 \%)$ was slightly higher than VC.

(2) Scavenging Ability of Superoxide Anion Radicals on EPS. On the contrary, un-deproteinized EPS showed better ability to scavenge superoxide anion radicals than deproteinized EPS (Figure 8). The scavenging rate increased significantly when the concentration of EPS was in the range of $0-2 \mathrm{mg} /$ $\mathrm{mL}$ and slowly at a concentration range from 2 to $10 \mathrm{mg} / \mathrm{mL}$.

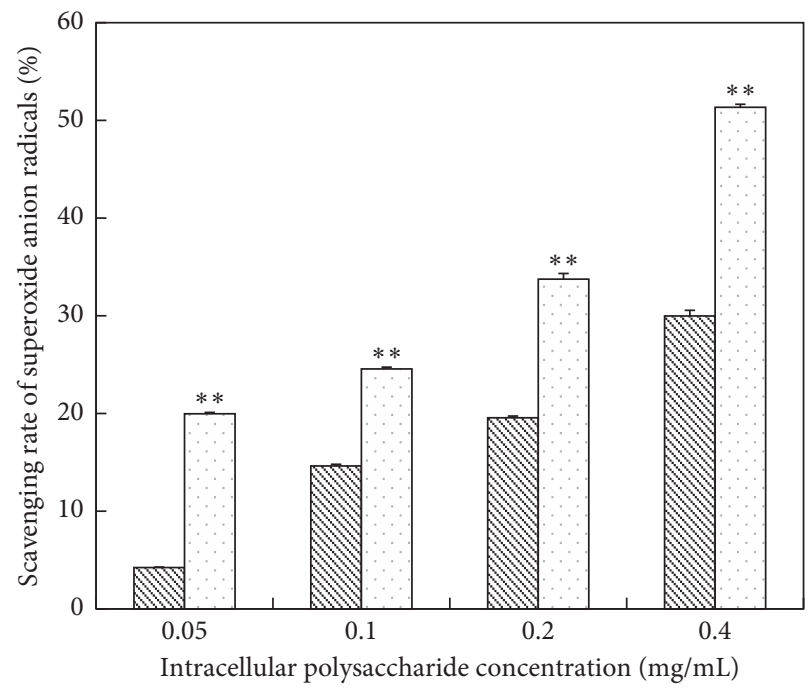

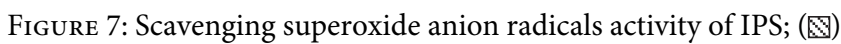
un-deproteinized IPS; ( $\square$ ) deproteinized IPS. The significance in difference between un-deproteinized IPS and deproteinized IPS was calculated by the paired-samples $T$ test $\left({ }^{* *} P<0.01\right)$.

At a concentration of $2 \mathrm{mg} / \mathrm{mL}$, the scavenging rate was $6.2 \%$ (the un-deproteinized), 2.2\% (the deproteinized) while the scavenging rate reached $11.1 \%$ (un-deproteinized EPS) and $6.3 \%$ (deproteinized EPS) at a concentration of $10 \mathrm{mg} / \mathrm{mL}$, respectively. Moreover, the scavenging ability of EPS on superoxide anion radicals was obviously lower than that of the positive controls (Table 5).

\subsubsection{Reducing Power Determination}

(1) Determination of Reducing Power of IPS. Reducing power value of IPS appeared in two manners with increased concentrations (Figure 9). In a certain range of polysaccharides concentration, the reducing power of deproteinized IPS was stronger than un-deproteinized IPS. At a concentration of $0.2 \mathrm{mg} / \mathrm{mL}$, the reducing power of deproteinized IPS was 0.044 while that of un-deproteinized IPS was 0.032 . Instead, the reducing power of deproteinized IPS was 0.088 at a concentration of $0.4 \mathrm{mg} / \mathrm{mL}$, while that of un-deproteinized IPS was a little higher (0.09). However, at a concentration of $0.05 \mathrm{mg} / \mathrm{mL}$, the antioxidant ability was too small to be detected because of the lower yield. In the mass, the reducing power of $\mathrm{VC}$ was clearly higher than that of undeproteinized IPS and deproteinized IPS (Table 6).

(2) Determination of Reducing Power of EPS. As the concentration of the EPS was increased, the reduction power of the deproteinized and un-deproteinized EPS was gradually 
TABLE 5: Scavenging superoxide anion radical activity of VC.

\begin{tabular}{lccccc}
\hline \multirow{2}{*}{ Scavenging rate of superoxide anion radicals (\%) } & \multicolumn{4}{c}{ VC concentration $(\mathrm{mg} / \mathrm{mL})$} \\
& 0.1 & 0.2 & 0.4 & 0.6 & 0.8 \\
\hline VC & $25.1 \pm 1.5$ & $31.4 \pm 0.79$ & $40.5 \pm 0.83$ & $49.3 \pm 0.94$ & $55.6 \pm 0.67$ \\
\hline
\end{tabular}

Each value is a mean of three biological replicates. Data shown are mean \pm standard deviation.

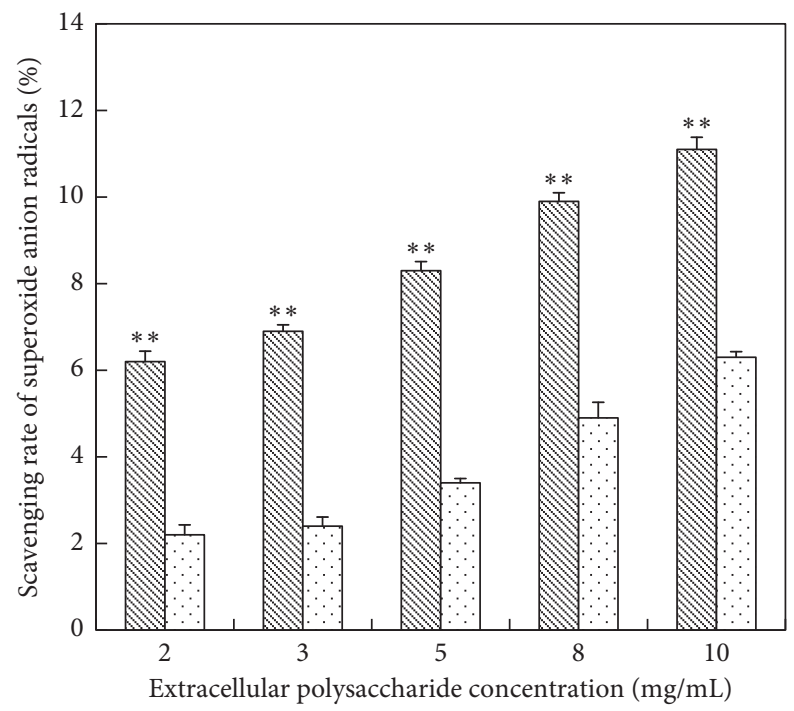

FIGURE 8: Scavenging superoxide anion activity of EPS; (专) un-deproteinized EPS; $\square$ ) deproteinized EPS. The significance in difference between un-deproteinized EPS and deproteinized EPS was calculated by the paired-samples $T$ test $\left({ }^{* *} P<0.01\right)$.

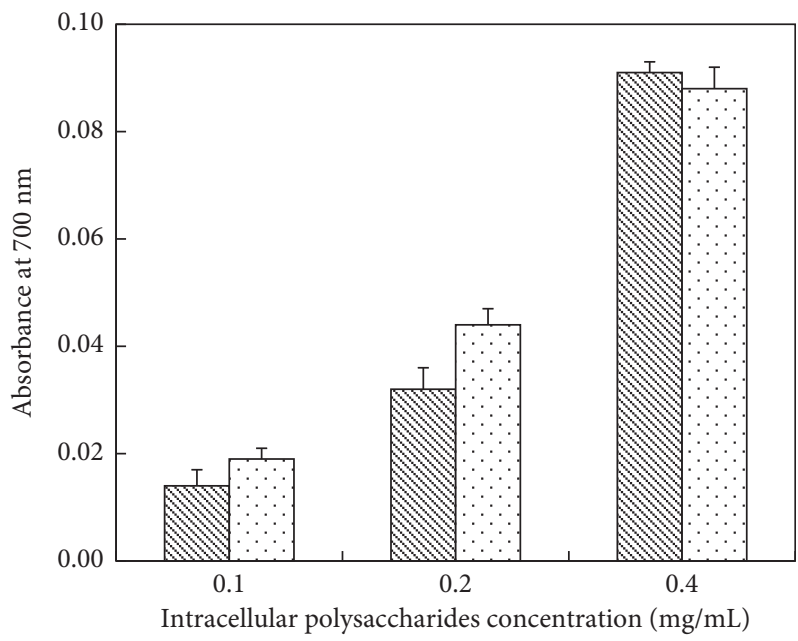

FIGURE 9: Reducing power of IPS; (图) un-deproteinized IPS; ( $\square$ ) deproteinized IPS. The significance in difference between un-deproteinized IPS and deproteinized IPS was calculated by the paired-samples $T$ test $\left({ }^{* *} P<0.01\right)$.

TABLE. 6: Reducing power of VC.

\begin{tabular}{lccccc}
\hline \multirow{2}{*}{ Absorbance at $700 \mathrm{~nm}$} & \multicolumn{4}{c}{ VC concentration $(\mathrm{mg} / \mathrm{mL})$} \\
& 0.1 & 0.2 & 0.4 & 0.6 & 0.8 \\
\hline VC & $0.23 \pm 0.01$ & $0.27 \pm 0.02$ & $0.3 \pm 0.15$ & $0.33 \pm 0.01$ & $0.38 \pm 0.02$ \\
\hline
\end{tabular}

Each value is a mean of three biological replicates. Data shown are mean \pm standard deviation. 


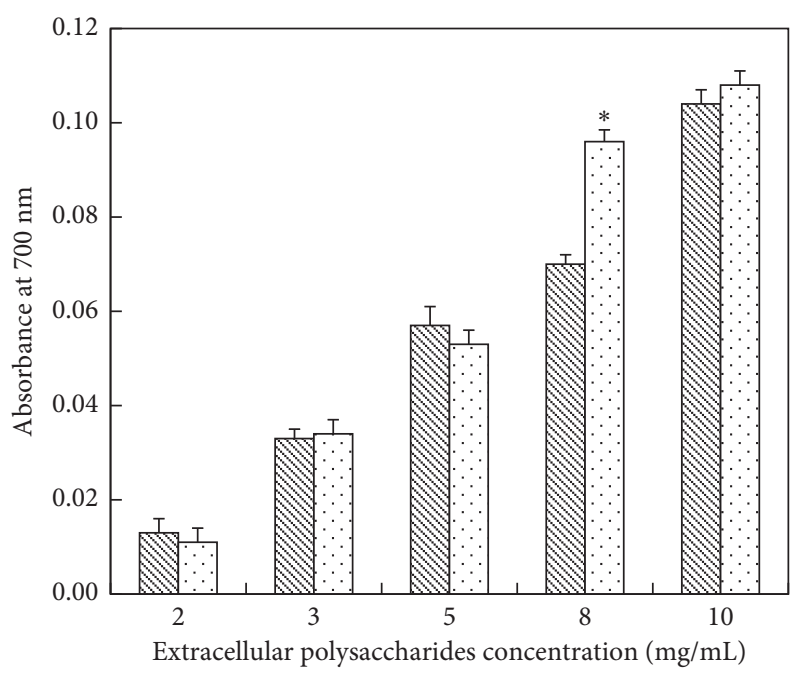

FIGURE 10: Reducing power of EPS; ( $\mathbb{S}$ ) un-deproteinized EPS; $(\square)$ deproteinized EPS. The significance in difference between undeproteinized EPS and deproteinized EPS was calculated by the paired-samples $T$ test $\left({ }^{*} P<0.05\right)$.

enhanced (Figure 10). At a concentration range from $0 \mathrm{mg} /$ $\mathrm{mL}$ to $5 \mathrm{mg} / \mathrm{mL}$, there was little difference in the reducing power between two kinds of EPS. At a concentration of $8 \mathrm{mg} / \mathrm{mL}$, the reducing power of the deproteinized (0.096) and un-deproteinized (0.07) EPS was significantly different. At a concentration range from $6 \mathrm{mg} / \mathrm{mL}$ to $10 \mathrm{mg} / \mathrm{mL}$, the reducing power of un-deproteinized EPS was weaker than that of the deproteinized protein. At $10 \mathrm{mg} / \mathrm{mL}$, the reducing powers of deproteinized and un-deproteinized EPS were 0.104 and 0.108 , respectively. Compared with VC positive control, the reducing powers of un-deproteinized and deproteinized EPS were wesker (Table 6).

\subsubsection{DPPH Radical-Scavenging Activity}

(1) Scavenging Ability of DPPH Free Radicals on IPS. The ability of deproteinized IPS on scavenging DPPH free radicals was stronger than that of un-deproteinized EPS (Figure 11). With regard to the un-deproteinized IPS, the scavenging rate was increased from $81.87 \%$ to $87.12 \%$, dramatically from $0.05 \mathrm{mg} / \mathrm{mL}$ to $0.4 \mathrm{mg} / \mathrm{mL}$. As for the deproteinized IPS, the scavenging rate reached $93.35 \%$ at a concentration of $0.1 \mathrm{mg}$ / $\mathrm{mL}$. However, lower concentration of VC demonstrated highest scavenging ability (Table 7).

(2) Scavenging Ability of DPPH Free Radicals on EPS. Obviously, there was no significant difference between undeproteinized EPS and deproteinized EPS (Figure 12). The scavenging rate of the un-deproteinized reached $92.6 \%$ while that of the deproteinized was $94.1 \%$ at a concentration of $10 \mathrm{mg} / \mathrm{mL}$. In general, the results showed that the scavenging ability of polysaccharides against DPPH free radicals was not as good as that of VC positive control (Table 7).

\section{Discussion}

Submerged cultivation is a promising and still underexplored alternative for the extraction of bioactive molecules in short time [22, 23], and large scale production under carefully monitored and controlled conditions is required for economic and scientific reasons [24]. According to the yield of polysaccharides, the optimized aeration rate for liquid fermentation was $4.5 \mathrm{~L} / \mathrm{min}$. The results showed that optimal aeration rate was helpful for polysaccharides yield of Hygrophoropsis sp., resulting in sufficient oxygen supply, which is frequently found in other fungal fermentation [25-27].

The results of antioxidation showed that the ability of reducing power, scavenging superoxide anion, and scavenging DPPH free radicals of deproteinized IPS was stronger than that of un-deproteinized IPS. However, the ability of IPS on scavenging DPPH radicals was lower than that of VC control. The ability of scavenging hydroxyl radicals was weaker than that of the un-deproteinized. These antioxidant capabilities are all considered to mainly be linked with the polysaccharides components of the extracts, resulting in significant medicinal efficacy, thus supporting the idea of using such compounds as active ingredients in functional products.

Though peptide from extracts showed antioxidant and anticancer activities, the extracts containing impurities like proteins may greatly limit their application in the fields of medicine and food, such as serious allergic reactions [28-30]. But proteins cannot be completely removed due to the intensive binding of some proteins to polysaccharides and the existence of glucoprotein or proteoglycan [31]. Here, deproteinized extracts showed stronger antioxidant activities than those of un-deproteinized in three assays tested, which demonstrated efficiency extracting method making little loss for bioactivity or protein making no effort for antioxidant activities.

The components having antioxidation activity in Hygrophoropsis sp. are unknown. As demonstrated here, polysaccharide could be one of the candidates that possessed the antioxidation activity; at least, such activity was enriched along together with the enrichment of polysaccharide in 


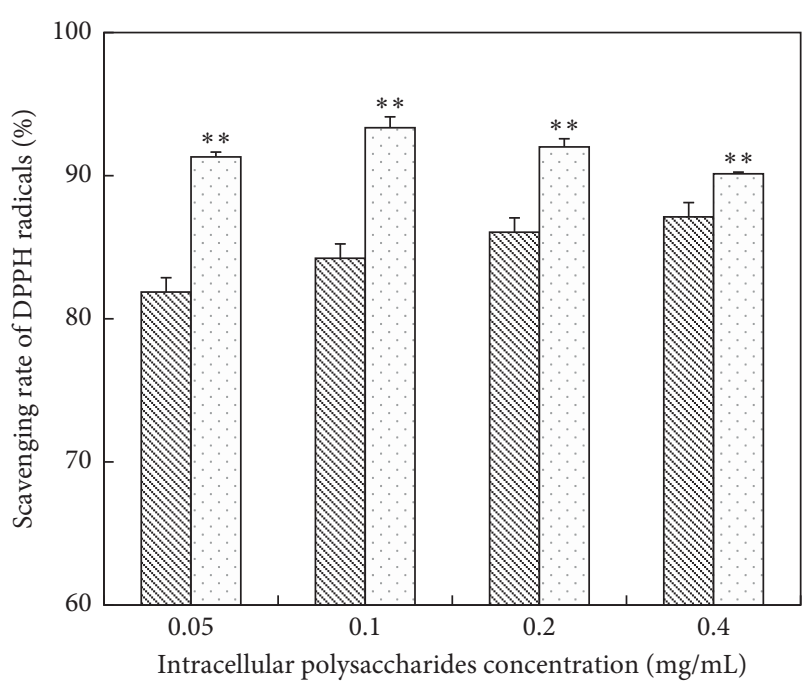

FIGURE 11: Scavenging DPPH free radicals activity of IPS; (圆) undeproteinized IPS; ([.) deproteinized IPS. The significance in difference between un-deproteinized IPS and deproteinized IPS was calculated by the paired-samples $T$ test $\left({ }^{* *} P<0.01\right)$.

TABLE 7: Scavenging DPPH free radicals activity of VC.

\begin{tabular}{ccccc}
\hline $\begin{array}{c}\text { VC concentration } \\
(\mathrm{mg} / \mathrm{mL})\end{array}$ & 0.05 & 0.1 & 0.2 & 0.4 \\
\hline
\end{tabular}

Scavenging rate of

DPPH radicals $\quad 94.7 \pm 0.87 \quad 95.4 \pm 0.72 \quad 95.7 \pm 0.85 \quad 96 \pm 0.67$ (\%)

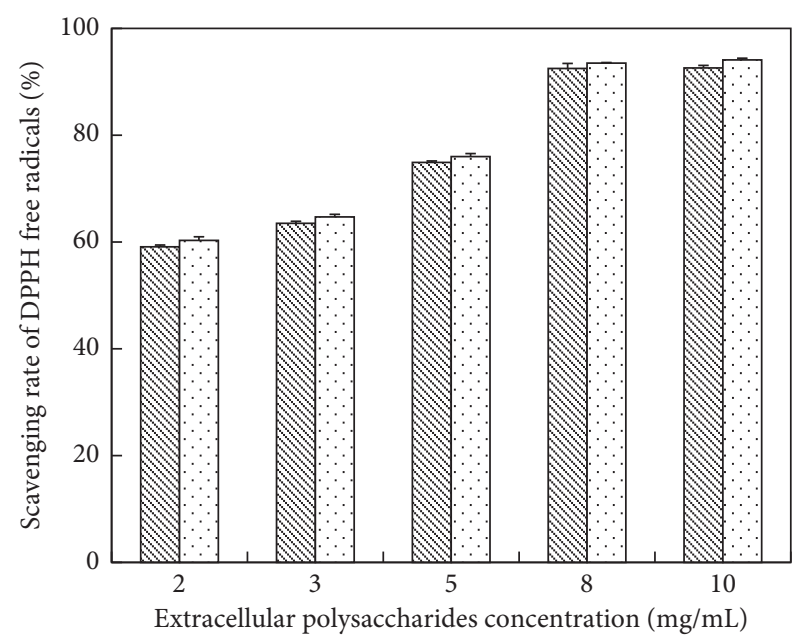

FIGURE 12: Scavenging DPPH free radical activity of EPS; (\$) undeproteinized EPS; ( $\square$ ) deproteinized EPS. The significance in difference between un-deproteinized EPS and deproteinized EPS was calculated by the paired-samples $T$ test $\left({ }^{*} P<0.05\right)$.

water extract derived from cultured Hygrophoropsis sp. mycelia. However, the exact identity of polysaccharide having antioxidation activity is not known. Additionally, the antioxidation activity could be due to other constituents that were enriched together with polysaccharides. At present, we are trying to purify further the enriched polysaccharides and to evaluate the identity of the active compound. Since by removing the bulk of polysaccharides from cultured Hygrophoropsis sp. mycelia the antioxidant activity (data not shown) was lost, it can be suggested that the polysaccharide is the key component exhibiting the antioxidation activity at least in part. This result is the same as Cordyceps [1]. By enrichment of the polysaccharide from cultured Hygrophoropsis sp. mycelia, the grade of antioxidation activities obtained was different, which suggested that several forms of polysaccharides might have different potency in the tested assays.

Compared with the deproteinized EPS, the ability of undeproteinized EPS on scavenging hydroxyl radicals and superoxide anion was increased, while the un-deproteinized EPS was less effective in the reducing power and scavenging $\mathrm{DPPH}$ free radicals. In addition, the ability of EPS to scavenge $\mathrm{DPPH}$ radicals (weaker than that of the VC control) is the strongest. The results showed that the protein in fermentation liquid extracts had a certain effect on the oxidation resistance of the polysaccharides. This result is the same as polysaccharide-protein complex (PSK) that has been obtained from the mycelium of Coriolus versicolor with a higher share of proteins, which were more effective in antioxidative activity to scavenge superoxide and hydroxyl radicals [32].

The IPS had higher antioxidation activities than EPS at the same amount in all the assays tested. Differences in molecular level and monosaccharide composition between IPS and EPS may be the reason that the antioxidant differences existed between them [33]. Many works of the literature reported moderate correlation between the reducing ability of $\mathrm{Fe}^{3+}$ and the total protein content, which may be a consequence of the presence of reductive amino acids of cysteine, methionine, and tyrosine in proteins of the extract $[34,35]$. In present study, neither deproteinized EPS nor deproteinized IPS showed more effective chelators of ferrous ions than un-deproteinized polysaccharides. Thus, the amino acids in the extracts of Hygrophoropsis sp. may be the further interesting research.

In conclusion, the water extracts of cultured Hygrophoropsis sp. mycelia could be chosen for natural additives of functional products. This is the first study known to evaluate the antioxidant capacity of deproteinized and un-deproteinized extracts from mushroom mycelium. However, the relevance of these in vitro results must be supported by future in vivo studies and whether the protein part of the extract in allergens should be demonstrated especially.

\section{Data Availability}

The data used to support the findings of this study are available from the corresponding author upon request.

\section{Conflicts of Interest}

The authors declare that they have no conflicts of interest.

\section{Authors' Contributions}

Liang Huang and Chunxia Li contributed equally to this study. LH and CL conducted research work and drafted 
manuscript. NS contributed to experimental design and data analysis. YW participated in editing of the manuscript. HY and YL assisted in conducting experimental trials. LB supervised all experimental trials and reviewed manuscript. All authors read and approved the final manuscript.

\section{Acknowledgments}

This work was financially supported by the Scientific Research Plan of Tianjin Educational Committee (20140607), the Innovation Team of Tianjin Vegetables Research System (ITTVRS2017015), the Tianjin Municipal Special Program of Talents Development for Excellent Youth Scholars (TJTZJHQNBJRC-2-12), and Tianjin Major special project of seed industry science and Technology (16ZXZYNC00210).

\section{References}

[1] S. P. Li, P. Li, T. T. X. Dong, and K. W. K. Tsim, “Antioxidation activity of different types of natural Cordyceps sinensis and cultured Cordyceps mycelia," Phytomedicine, vol. 8, no. 3, pp. 207-212, 2001.

[2] I. C. F. R. Ferreira, S. A. Heleno, F. S. Reis et al., "Chemical features of Ganoderma polysaccharides with antioxidant, antitumor and antimicrobial activities," Phytochemistry, vol. 114 , pp. 38-55, 2015.

[3] C. G. C. Rúbia, A. H. P. D. Souza, R. C. Calhelha et al., "Bioactive formulations prepared from fruiting bodies and submerged culture mycelia of the Brazilian edible mushroom Pleurotus ostreatoroseus singer," Food \& Function, vol. 6, no. 7, pp. 2155-2164, 2015.

[4] V. Emanuel, "Antioxidant properties of mushroom mycelia obtained by batch cultivation and tocopherol content affected by extraction procedures," Biomed Research International, vol. 2014, Article ID 974804, 2014.

[5] M. Kozarski, A. Klaus, M. Niksic, G. van, M. Vrvic, and D. Jakovljevic, "Polysaccharides of higher fungi: biological role, structure, and antioxidative activity," Chemical Industry, vol. 68, no. 3, pp. 305-320, 2014.

[6] Q. Wang, F. Wang, Z. Xu, and Z. Ding, "Bioactive mushroom polysaccharides: a review on monosaccharide composition, biosynthesis and regulation," Molecules, vol. 22, no. 6, pp. 955-968, 2017.

[7] J. P. F. Angeli, L. R. Ribeiro, M. L. C. Gonzaga et al., "Protective effects of $\beta$-glucan extracted from Agaricus brasiliensis against chemically induced DNA damage in human lymphocytes," Cell Biology and Toxicology, vol. 22, no. 4, pp. 285-291, 2006.

[8] T. G. Pillai, C. K. K. Nair, and K. K. Janardhanan, "Enhancement of repair of radiation induced DNA strand breaks in human cells by Ganoderma mushroom polysaccharides," Food Chemistry, vol. 119, no. 3, pp. 1040-1043, 2010.

[9] X.-L. Liu, X.-Q. Zheng, P.-Z. Qian et al., "Purification and characterization of a novel fibrinolytic enzyme from culture supernatant of Pleurotus ostreatus," Journal of Microbiology and Biotechnology, vol. 24, no. 2, pp. 245-253, 2014.

[10] B.-Z. Zhang, K. T. Inngjerdingen, Y.-F. Zou et al., "Characterisation and immunomodulating activities of exo-polysaccharides from submerged cultivation of Hypsizigus marmoreus," Food Chemistry, vol. 163, pp. 120-128, 2014.

[11] T. L. Tao, F. J. Cui, and X. X. Chen, "Improved mycelia and polysaccharide production of Grifola frondosa by controlling morphology with microparticle Talc," Microbial Cell Factories, vol. 17, no. 1, pp. 1-10, 2018.
[12] S. Zhang, X. Liu, L. Yan, and Q. Zhang, "Chemical compositions and antioxidant activities of polysaccharides from the sporophores and cultured products of Armillaria mellea," Molecules, vol. 20, no. 4, pp. 5680-5697, 2015.

[13] J. Huang, H.-J. Zhang, and C.-P. Xu, "Culture characterization of exopolysaccharides with antioxidant activity produced by Pycnoporus sanguineus in stirred-tank and airlift reactors," Journal of the Taiwan Institute of Chemical Engineers, vol. 45, no. 5, pp. 2075-2080, 2014.

[14] G. Huang, Q. Yang, and Z. B. Wang, "Extraction and deproteinization of mannan oligosaccharides," Zeitschrift für Naturforschung C, vol. 65, no. 5-6, pp. 387-390, 2010.

[15] S. Xu, Y. Zhang, and K. Jiang, "Antioxidant activity in vitro and in vivo of the polysaccharides from different varieties of Auricularia auricula," Food \& Function, vol. 7, no. 9, pp. 3868-3879, 2016.

[16] M. Dubois, K. A. Gilles, J. K. Hamilton, P. A. Rebers, and F. Smith, "Colorimetric method for determination of sugars and related substances," Analytical Chemistry, vol. 28, no. 3, pp. 350-356, 1956.

[17] S.-I. Lim, C.-W. Cho, U.-K. Choi, and Y.-C. Kim, “Antioxidant activity and ginsenoside pattern of fermented white ginseng," Journal of Ginseng Research, vol. 34, no. 3, pp. 168-174, 2010.

[18] S. Marklund and G. Marklund, "Involvement of the superoxide anion radical in the autoxidation of pyrogallol and a convenient assay for superoxide dismutase," European Journal of Biochemistry, vol. 47, no. 3, pp. 469-474, 1974.

[19] M. Oyaizu, "Studies on products of browning reaction. antioxidative activities of products of browning reaction prepared from glucosamine," The Japanese Journal of $\mathrm{Nu}$ trition and Dietetics, vol. 44, no. 6, pp. 307-315, 1986.

[20] T.-H. Kao and B.-H. Chen, "Functional components in soybean cake and their effects on antioxidant activity," Journal of Agricultural and Food Chemistry, vol. 54, no. 20, pp. 7544-7555, 2006.

[21] L. Ming, T. Lei, and C. Shuliang, "Design method of controllable blade angle and orthogonal optimization of pressure rise for a multiphase pump," Energies, vol. 11, no. 5, pp. 1048-1068, 2018.

[22] A. Zilly, A. Bracht, J. S. Coelho-Moreira et al., "Enzymes from Ganoderma lucidum: production and applications," Current Topics in Biochemical Research, vol. 13, pp. 1-11, 2011.

[23] R. C. G. Corrêa, T. Brugnari, A. Bracht, and M. R. Peralta, "Biotechnological, nutritional and therapeutic uses of Pleurotus spp. (oyster mushroom) related with its chemical composition: a review on the past decade findings," Trends in Food Science \& Technology, vol. 50, pp. 103-117, 2016.

[24] R. J. Seviour, B. Mcneil, M. L. Fazenda, and L. M. Harvey, "Operating bioreactors for microbial exopolysaccharide production," Critical Reviews in Biotechnology, vol. 31, no. 2, pp. 170-185, 2011.

[25] Y. Dou, J.-H. Xiao, X.-X. Xia, and J.-J. Zhong, "Effect of oxygen supply on biomass and helvolic acid production in submerged fermentation of Cordyceps taii," Biochemical Engineering Journal, vol. 81, pp. 73-79, 2013.

[26] H. H. Kim, J.-G. Na, Y. K. Chang, and S. J. Lee, "Effects of dissolved oxygen control on cell growth and exopolysaccharides production in batch culture of Agaricus blazei," Korean Journal of Chemical Engineering, vol. 22, no. 1, pp. 80-84, 2005.

[27] Z.-H. Wei, N. Chen, Y.-J. Li et al., "Glucose fed-batch integrated dissolved oxygen control strategy enhanced polysaccharide, total triterpenoids and inotodiol production in 
fermentation of a newly isolated Inonotus obliquus strain," Process Biochemistry, vol. 66, pp. 1-6, 2018.

[28] X. Zeng, P. Li, X. Chen et al., "Effects of deproteinization methods on primary structure and antioxidant activity of Ganoderma lucidum polysaccharides," International Journal of Biological Macromolecules, vol. 126, pp. 867-876, 2019.

[29] S. Frémont, C. Sanchez, and Y. Errahali, "Food allergy: effect of proteins-lipids and proteins-polysaccharides interactions," European Annals of Allergy \& Clinical Immunology, vol. 36, no. 3, pp. 82-87, 2004.

[30] Z. Chen, W. Li, R. K. Santhanam et al., "Bioactive peptide with antioxidant and anticancer activities from black soybean [Glycine max (L.) Merr.] byproduct: isolation, identification and molecular docking study," European Food Research and Technology, vol. 245, no. 3, pp. 677-689, 2019.

[31] Y. Chen, M. Xie, W. Li et al., "An effective method for deproteinization of bioactive polysaccharides extracted from lingzhi (Ganoderma atrum)," Food Science and Biotechnology, vol. 21, no. 1, pp. 191-198, 2012.

[32] F. Liu, V. E. C. Ooi, and S. T. Chang, "Free radical scavenging activities of mushroom polysaccharide extracts," Life Sciences, vol. 60, no. 10, pp. 763-771, 1997.

[33] Y.-H. Jiang, X.-L. Jiang, P. Wang, and X.-K. Hu, “In vitro antioxidant activities of water-soluble polysaccharides extracted from Isaria farinosa b05," Journal of Food Biochemistry, vol. 29, no. 3, pp. 323-335, 2005.

[34] M. Kozarski, A. Klaus, M. Niksic, D. Jakovljevic, J. P. F. G. Helsper, and L. J. L. D. Van Griensven, "Antioxidative and immunomodulating activities of polysaccharide extracts of the medicinal mushrooms Agaricus bisporus, Agaricus brasiliensis, Ganoderma lucidum and Phellinus linteus," Food Chemistry, vol. 129, no. 4, pp. 1667-1675, 2011.

[35] M. Kozarski, Chemical characterization, antioxidative and antimicrobial properties of polysaccharide extracts from the selected mushroom species, Ph.D. thesis, Faculty of Chemistry, University of Belgrade, Belgrade, Serbia, 2012. 